\title{
Exploring the Focal Role of Pyroptosis in Diabetes Mellitus
}

\author{
Ishita Sharma ${ }^{1}(\mathbb{D})$, Tapan Behl ${ }^{1, *}{ }^{\mathbb{D}}$, Aayush Sehgal ${ }^{1}$ (D), Sukhbir Singh 1 (D), Neelam Sharma ${ }^{1}$ (D), \\ Harmanpreet Singh 1 (D), Abdul Hafeez ${ }^{2}$ (D), Simona Bungau 3 (D) \\ Chitkara College of Pharmacy, Chitkara University, Punjab, India \\ 2 Glocal School of Pharmacy, Glocal University, Mirzapur Pole, Saharanpur, Uttar Pradesh, India \\ 3 Department of Pharmacy, Faculty of Medicine and Pharmacy, University of Oradea, Romania \\ * Correspondence: tapanbehl31@gmail.com (T.B);
}

Scopus Author ID: 56560241200

Received: 11.01.2021; Revised: 6.02.2021; Accepted: 8.02.2021; Published: 13.02.2021

\begin{abstract}
Diabetes mellitus is a T cell-mediated disease associated with the depletion of beta cells responsible for insulin production. Although the disease is $\mathrm{T}$ cell-mediated, it undergoes various biochemical responses and programmed cell death. Programmed cell death, a distinct biochemical pathway in which cells die by eliciting various physiological outcomes. Pyroptosis, apoptosis, and necrosis are the three major forms of programmed cell death that function as a defense mechanism against various infections, diseases, and microorganisms. This review article focuses on the various pathological mechanisms of pyroptosis. Pyroptosis is distinguished by the caspase-1-dependent formation of plasma membrane pores, resulting in the release of pro-inflammatory cytokines, leading to cell lysis. Caspase-1, a protease which is an interleukin-1L-1 $\beta$ converting enzyme that initiates the cell death process by converting interleukin-1L-1 $\beta$ into mature inflammatory cytokine (mature form). Emerging evidence has made pyroptosis a vital trigger as well as an endogenic regulator of diabetes mellitus.
\end{abstract}

Keywords: pyroptosis; diabetes mellitus; caspase-1; inflammasomes; interleukin-1L-1 $\beta$.

(C) 2021 by the authors. This article is an open-access article distributed under the terms and conditions of the Creative Commons Attribution (CC BY) license (https://creativecommons.org/licenses/by/4.0/).

\section{Introduction}

Cells can die through an assortment of biochemical pathways by eliciting various physiological outcomes. As different biological pathways program their death, this phenomenon is also known as programmed cell death [1]. Cell death plays a vital role in the development and endurance of an organism. Pyroptosis and apoptosis are examples of programmed cell death, and both are executed by caspase. Caspase-1, a protease known to be an interleukin-1L-1beta converting enzyme, converts interleukin 1beta into its mature form, i.e., in mature inflammatory cytokines [2-5]. So, the activation of protease caspase-1 leads to the formation of inflammatory cytokines and ultimately accounts for cell death via the plasma membrane's rupturing [6-9]. Apoptosis, a silent process that elicits its action in the defense mechanism against infection. Whereas pyroptosis, stimulated by most microbial and noninfectious diseases, is caused by an inflammatory caspase [2,3].

Moreover, the host factors, i.e., angina pectoris and hypertension in myocardial infarction, play an essential role in pyroptosis functioning [3,7]. The death that is dependent on the cell is profound to be programmed self-destruction and hence, regulated by caspase. Thus, pyroptosis is defined as inherited programmed death regulated by caspase. Hence, it has the 
characteristics of both apoptosis and necrosis [10,11]. Henceforth, pyroptosis leads to cytokines' release and further activates inflammatory mediators of cell [12]. Also, pyroptosis, a lytic, features cell swelling and elicits large bubbles from the plasma membrane [12-14].

Diabetes mellitus is a complication associated with depletion in the beta cells producing insulin. It is a cluster of metabolic disorders that widely acclaim hyperglycemia, glycosuria, negative nitrogen balance attributed to defect in the secretion of insulin in the body. Long-term hyperglycemia accounts for distinct side effects that comprise organ failure, dysfunction of organs, etc. Various other processes that are being involved in diabetes development are; disruption of pancreatic beta cells, deficiency of insulin that directly cause resistance in insulin action. The above' cumulative action is seen in carbohydrates, fats, and protein metabolism abnormalities in patients with diabetes. Symptoms associated with hyperglycemia include ketoacidosis, weight loss, blurred vision, and polyphagia [15-19].

Hyperglycemia has an intricate role, and its effect is seen in the long-term as various complications. These complications are related to the eyes, nerves, and kidneys and are commonly termed retinopathy, neuropathy, and nephropathy. Retinopathy, a complication in the eyes due to diabetes, is the leading cause of blurred vision. Nephropathy leads to renal failure. Neuropathy is related to foot ulcers and Charcot joints. Its incidence even encompasses abnormalities in the heart, including hypertension, atherosclerosis, and peripheral artery diseases [20,21].

Diabetes mellitus falls into two broad categories: Type-1 diabetes mellitus, i.e., insulindependent, and Type- 2 diabetes mellitus, i.e., non-insulin-dependent. Type- 1 is attributed to the disruption of beta cells present in the pancreatic islets. Most of the disruption is related to autoimmune antibodies, and destroyed beta cells are easily noticeable in blood, but no antibody is detected. Whereby, in type-2 also no beta-cell antibody is detected in the blood. Here, either the insulin found is low in circulation or is too high or normal, depending on the integrity of diabetes. Over $90 \%$ of cases of diabetes are known to be type-2. The main cause of type- 2 diabetes is an abnormality found in the glucoreceptors of beta cells, leading to progression in insulin secretion impairment [15]. Diabetes is also developed by inflammasomes, i.e., a multiprotein complex that detects pathogens, microorganisms in the body. These, in response to pathogens, activate pro-inflammatory cytokines interleukin-1L-1beta and interleukin-1L-18. These interleukins are generated from the secretion of caspase-1, which further leads to pyroptosis, i.e., cell death, in patients with diabetes mellitus.

The nucleotide-binding domain (NOD receptors) was the first one discovered that niche as forming up inflammasome [21,22]. NLR is being classified according to the domain structure, as all the NLR contain LRR (leucine-rich repeat), nucleotide-binding domain (NBD), and a signal domain [23]. Signal domain, executes the caspase-1 recruitment by CARD or by pyrin domain. This whole process leads to the death of a cell in diabetic patients. It consists of an adaptor molecule apoptosis-associated speck-like protein containing a CARD (ASC) [24]. The assembly of inflammasome triggers out in response to stimulus found during any infection or cellular stress that in return activates caspase-1. The first family of sensor proteins discovered was NOD-like receptors; these receptors are efficient enough to form inflammasomes. It constitutes a signaling domain. It further plays a vital role in the activation of caspase-1 [25,26]. Signaling domain causes expression of caspase-1 through CARD and pyrin domain. CARD is considered an effector domain of ASC. PYRIN domain role's role is to regulate CARD oligomerization status by upstreaming signaling molecules in the activation pathway of caspase- 1 . The CARD and pyrin domain then allow the binding of capsase- 1 to 
itself. Inflammasomes are divided into; SNLRP1, NLRC4, AIM2 and NLRP3 [27,28]. NLRP3 inflammasome is the most intensely studied inflammasome, usually gets activated in all the diseases.

\section{Pathogenesis of Diabetes Mellitus}

Progression of the disease is known as pathogenesis, and several pathological mediums contribute to diabetes progression. Diabetes occurs due to beta islet cell death that produces insulin in the body. Apart from this, beta-cell death in diabetes occurs due to lymphocytes, CD4, macrophages, interleukins. In type-1 diabetes mellitus, heterogeneity of pancreatic lesions makes the pathogenesis a bit difficult to follow. Lymphocytic infiltration, found in islet beta cells, this heterogeneity of cells is being seen in diabetes-1. Apart from this, activation of CD4 T cells is a prerequisite in the development of diabetes. These cells are prone to produce diabetes, while CD8 T cells damage the diabetic patient $[28,29]$.

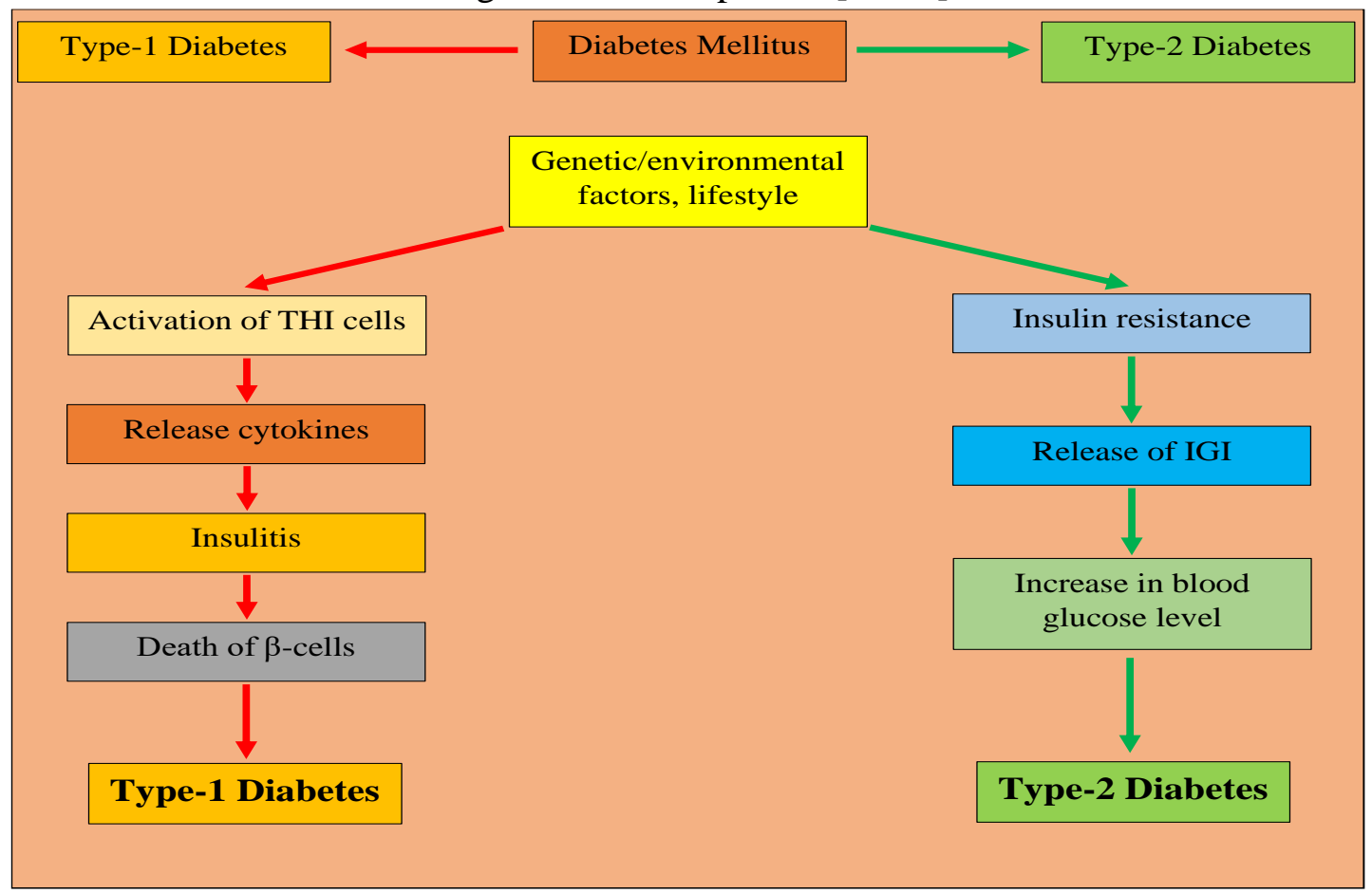

Figure 1. Pathogenesis of diabetes mellitus 1 and diabetes mellitus 2.

Islet cell proteins, presented by APC (antigen-presenting cells), express high levels of major histocompatibility complexes (MHC) molecules on their respective surfaces. These are highly active in the antigen presentation in autoimmune diabetes. APC generates interleukin cytokines, helps in promoting the differentiation of Th1 cells. These Th1 cells further stimulate the cytotoxic T cells, initiating the process of beta-cell death. The above discussed is the first step towards the initiation of diabetes disease. Interferons IFN-gamma secreted from T cells stimulate the action of resting macrophages to release cytokines $1 \mathrm{~L}-1$ beta and radicals. These cytokines and free radicals are considered to be toxic ones for pancreatic beta cells. Beta-cell damages are caused by the CD8 and T cells recognizing the autoantigen on beta cells [30,31]. Beta-cell damage resulted from the liberation of perforins. Thus, continuous destruction of beta cells eventually leads to diabetes [29].

Type-2 diabetes, most common in developing countries, generally affects 40-60 years old aged people. This is mainly caused by lifestyle changes, obesity, high fat diet, and lack of exercise. Diabetes -2 is mainly recognized by the need for insulin in the body that may cause 
ketoacidosis. Patients with diabetes-2 experience dysfunction of impaired beta cells and insulin resistance in the body [28].

Pathogenesis of type-2 diabetes involves the progression of insulin resistance in the body undergoing hyperinsulinemia and further progress in impairment of beta cells (Figure 1) [32]. Patients with type-2 diabetes face overproduction of glucose and low glycogenolysis. Release of inflammatory markers is subjected to be seen from the adipose tissues in the patients undergoing insulin resistance. The glucose, GLP-1 (glucagon-like peptide), fat hormones are the one that mediates signals to beta cells so as they respond to insulin resistance [33]. Apart from this, obesity, hypertension, and dysglycemia are the other major cause for the development of type- 2 diabetes. In obesity, insulin resistance in the body can decrease if minimizing dietary intake and maximizing regular exercise. The impairment of adipose tissues is mainly caused by various genetic and environmental factors, causing obesity. Its dysfunction can occur in hypoxia, fat deposition, stress, or inflammatory process in the cells. All this causes insulin impairment. This results in diabetes mellitus [34].

\section{Biology of Pyroptosis}

Rupturing, cell secretion and swelling of the cell are considered some of the features of pyroptosis associated with various diseases. According to various caspase types, the pathway of pyroptosis is classified into the canonical pathway and non-canonical pathway.

\subsection{Canonical pathway.}

Inflammasomes are considered multi proteins that serve as a molecular switch in pyroptosis and contribute to diverse inflammatory diseases. Nod-like receptors (NLRs) [35] are one of these pyroptosis molecular switches and consist of NLRP1, NLRP3, NLRP7, and NLRC4. NLRP3 inflammasomes are associated with being linked intricately with the occurrence of pyroptosis and get activated by various stimuli [36-40]. Damage-associated molecular patterns (DAMP) and pathogen-associated molecular patterns (PAMP) are the stimuli by which the NLRP3 inflammasome gets activated. In return, its activation further activates the assembly of caspase- 1 that causes cleavage of pro-inflammatory cytokines, which cause pyroptosis [41-43].

\subsection{Non-canonical pathway.}

The non-canonical pathway is also termed as an independent pathway of pyroptosis that caspase- 4 and caspase- 5 arbitrate in human beings and caspase-11 present in mice. Caspase4,5,11 possess high affinity and is activated by binding to lipopolysaccharide (LPS) [44,45]. It was seen that gasdermin $\mathrm{D}$, a protein in humans that is encoded by the gsdm $\mathrm{d}$ gene, is a substrate of inflammatory caspase. Caspase-11 responds to LPS, cleave 53-kDa precursor in an inactive form of gsdm d to generate $\mathrm{N}$-terminal fragment of gsdm d gene in return, triggers the induction of pyroptosis [46]. Maturation of $g s d m d$ is mediated by caspase-11, which further contributes to the activation of caspase- 1 and the release of $1 \mathrm{~L}-1 \beta$. Caspase- 11 can also activate non-canonical NLRP3 inflammasome without the stimulation of LPS [47].

\section{Activation Mechanisms of Pyroptosis}

Pyroptosis is a form of cell death that varies in morphological nature from other cell deaths. A crucial feature of pyroptosis is caspase- 1 dependent, an enzyme that mediates the 
cell death process. As studied above, apoptosis is another type of cell death in which caspase3 and 6 are involved, and caspase- 1 does not affect it [48,49]. Pyroptosis mainly features the release of pro-inflammatory cytokines along with rapid rupturing of plasma membranes. Henceforth, cell lysis/death during pyroptosis is because of caspase-1 mediated processes. The one with plasma membrane pores of caspase- 1 dependent disperses the gradients of cellular ions, thus producing an elevation in osmotic pressure and swelling of cells, automatically resulting in cell lysis, releasing inflammatory intracellular content [50]. So, cells that die by pyroptosis show some variant features like, increase in the size of cells, cytoprotective agent glycine block ion influx in the eukaryotic cells that are damaged, and this automatically prevents the swelling of the cell during pyroptosis. During pyroptosis, the cleavage of DNA results from caspase-1 activated nuclease in an unidentified form. It does not produce an oligonucleosomal DNA pattern, a characteristic of apoptosis. Cell lysis and DNA cleavage are both considered as caspase-1-dependent and a feature of pyroptosis. The actin cytoskeleton is also observed in the cells that undergo pyroptosis, but the mechanism is unclear [51].

Besides maturation and secretion of $1 \mathrm{~L}-1 \beta$ and $1 \mathrm{~L}-18$ (pro-inflammatory cytokines), the inflammasome activation triggers the inflammatory cell death termed pyroptosis. Inflammatory cytokines are released by the pyroptosis induced cell death [52]. Proinflammatory cytokines can easily lead to inflammatory effects, and pyroptosis and proinflammatory cytokines are considered a potential inflammatory mechanism [53,54]. Moreover, pyroptosis is also considered a removal mechanism to kill infected macrophages and dendritic cells; the pathogenic bacteria can be easily cleared. For instance, the activated form of caspase-1 can clear Salmonella typhimurium and Legionella pneumophile by pyroptosis induction. Few pathogenic infections are controlled by co-management of pyroptosis and cytokine secretion of cytokine. The above mentioned showed that pyroptosis has an exclusive biological effect and also promotes inflammation [55].

\subsection{Gasdermin D: an executioner of pyroptosis.}

Pyroptosis, the most effective defense mechanism against bacteria, eliminates compromised cells, thus removing pathogens' protective niche. Gasdermin D was evolved, acting as an executioner of pyroptosis, by forming pores [56,57]. It is a 53-KDa substrate. It is a member of the family of conserved proteins that encompass gasdermin A, B, C, D, and DFNB59. Humans contain one replica of each one gasdermin. Gasdermin, present in humans, is composed of 242 amino acids, which are connected to a carboxy-terminal chain having 199 amino acids [58,59]. As there are 2 domains of amino acids, domain $\mathrm{C}$ and domain $\mathrm{N}$, gasdermin joins the $\mathrm{N}$ terminal domain, named PFD [60]. Whereas, $\mathrm{C}$ terminal domain is addressed as RD [56,61]. After cleavage, RD separates from PFD, thereby integrating PFD in the cell membrane $[56,57,62]$. Both RD and PFD interact by a linker. The linker's main role is to join both the domains at one end, whereas PFDs alpha 4 helix extends itself from the $\mathrm{C}$ domain and gets binds to RD. Thereby, the cleavage of linker occurs, leading to the dissociation of alpha 4 helix and henceforth releasing PFD from RD [56,61,63-65].

After the caspase activation, two events take place, the opening of gasdermin pores and membrane rupture. These only take place because of the number of pores present on the plasma membrane. Many pores automatically result in plasma membrane separation from the cortical cytoskeleton, thereby rupturing the membrane [66]. 


\subsection{Gasdermin D mediates pyroptosis by pore formation.}

The open-pore of gasdermin D promotes the breaking of permeability of barrier of the plasma membrane. The most cataclysmic effect is the disruption of potassium and sodium ions present across the plasma membrane. Usually, a cytosol has elevated levels of potassium and deceived levels of sodium $[67,68]$. Opening of pores ultimately causes potassium efflux from the cell counter-balanced further by cytosol that pulls cytosol into it (Figure 2). Similarly, the influx of sodium is there, leading to the elevation in its concentration. This gradually causes cell volume to increase. Regulatory volume decrease should be followed if the gasdermin pores are few. Likewise, if the plasma membrane's pores are small, pore containing membrane should patch with the exocytic membrane fusions [69].

Alternatively, if the pores are present in exceeding number then, cell volume ultimately increases. Due to this elevated cell volume level, the plasma membrane eventually distincts itself from the cortical cytoskeleton. After that, this causes rupturing of the membrane that releases the cytosolic content. This rupturing is termed pyroptosis. All the caspases related to inflammasome directly cleave this 53-KDa substrate, known as gasdermin D [70]. This cleavage automatically causes the generation of $31-\mathrm{KDa} \mathrm{N}$-terminal that further induces pyroptosis.

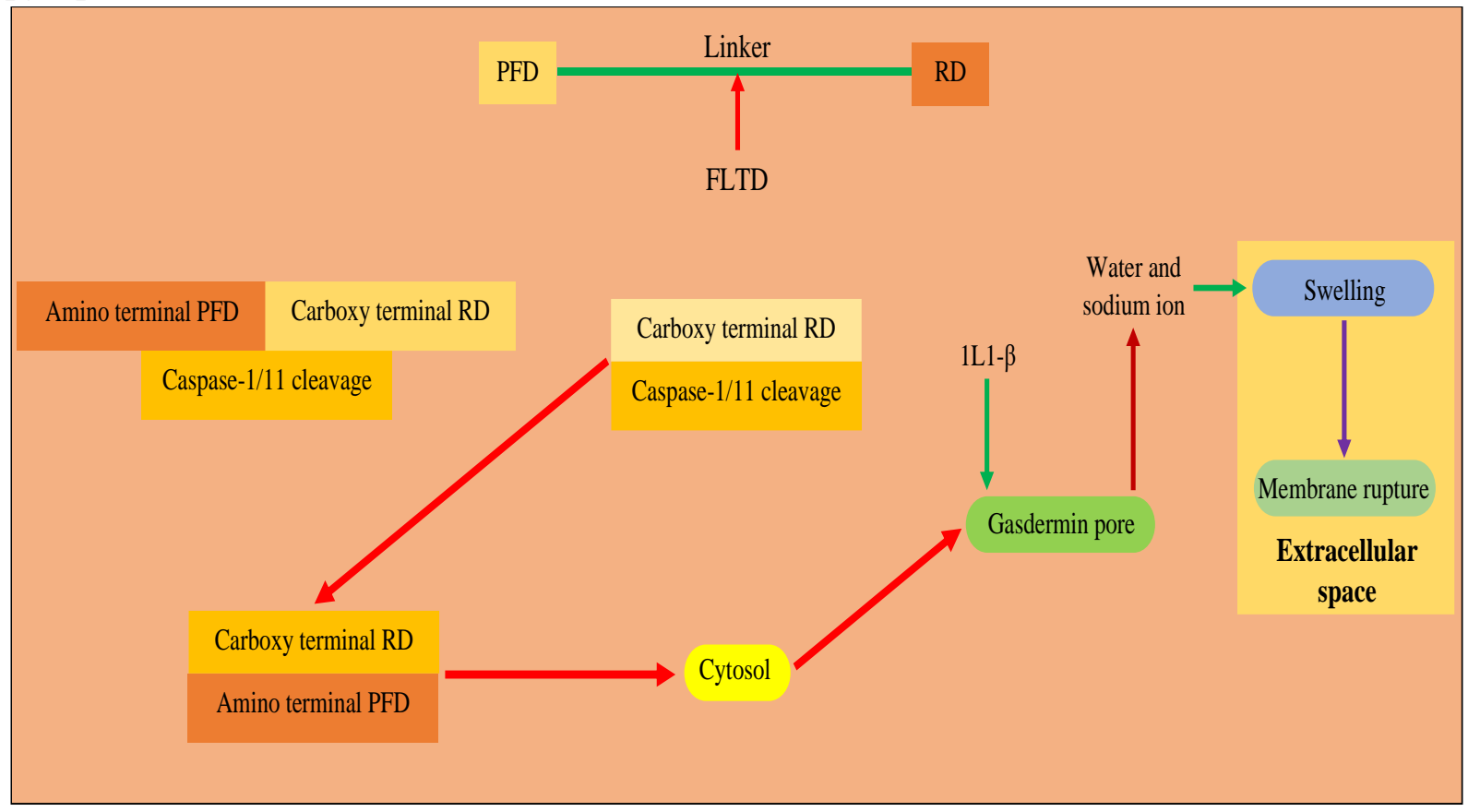

Figure 2. Role of gasdermin $\mathrm{D}$ in pyroptosis.

\section{Deciphering the Role of Inflammasomes and Pyroptosis in Diabetes Mellitus}

As studied above, pyroptosis is cell death, and inflammasomes act as a contributor to diabetes mellitus via pyroptosis by adding up to its progression via sensing various metabolic and oxidative stresses due to DM in the body [71-73]. Pyroptosis diabetes is commenced mainly via the activation of the NLRP3 inflammasome.

\subsection{ROS overproduction.}

Inflammasomes get activated in the diabetes mellitus via various mechanisms. Various stimulators of NLRP3 inflammasome induce ROS production, and elevation in ROS is responsible for activation of the inflammasome. ROS is known to be a result of mitochondrial 
oxidative phosphorylation. Through respiration, $2 \%$ of molecular oxygen is reduced to $\mathrm{OH}$ and superoxide, and oxygen production takes place at complex -1 and 3 in the mitochondrial respiratory chain. Complex-1 is when electrons are accepted from NADH that moves down an electrochemical gradient by ubiquinone to $3^{\text {rd }}$ complex. From complex-3, it moves down to complex-4 and cytochrome-C, when reduction of oxygen to water takes place [74].

Apart from mitochondrial electron transport, ROS is produced in mammalian cells by NADPH oxidase, lipoxygenase, and cyclooxygenase [75]. These proteins generate ROS by transferring electrons through various biological membranes to an electron acceptor via cytosolic donor (NADPH) in luminal space. ROS generation plays a vital role in cell signaling. Various physiological responses due to ROS may lead to pyroptosis, i.e., cell death. Production of ROS by polymorphonuclear leukocytes (PMN), exposed to priming via NLRP3 activators, then regulates NF-KB initiation, i.e., nuclear factor Kappa Light chain enhancer, and along with this, production of pro-inflammatory cytokines also takes place. Nlrp3 inflammasome plays a crucial role in promoting the processing of pro-inflammatory cytokines, i.e., $1 \mathrm{~L}-18$ and $1 \mathrm{~L}-1 \beta$, and activation of caspase-1 [76]. The diagrammatic representation depicts the secretion of pro-inflammatory cytokines due to metabolic stress (Figure 3). This depicts that danger signals are produced in the body due to any stress caused by a disease. Overproduction of ROS, endoplasmic stress and ATP production is known as metabolic stress for the body [77]. These are sensed by the signals DAMPs and PAMPs. The sensation would lead to the interaction of proteins within NLRP3, ASC, and procaspase-1 and further commence in the interaction of CARD-CARD, helpful in eliciting out caspase-1. The eliciting and activation of caspase-1 contribute to the secretion of pro-inflammatory cytokines, i.e., 1L-1beta and 1L-18. Secretions successfully contribute to the depletion of islets cells, causing pyroptosis, i.e., cell death [78].

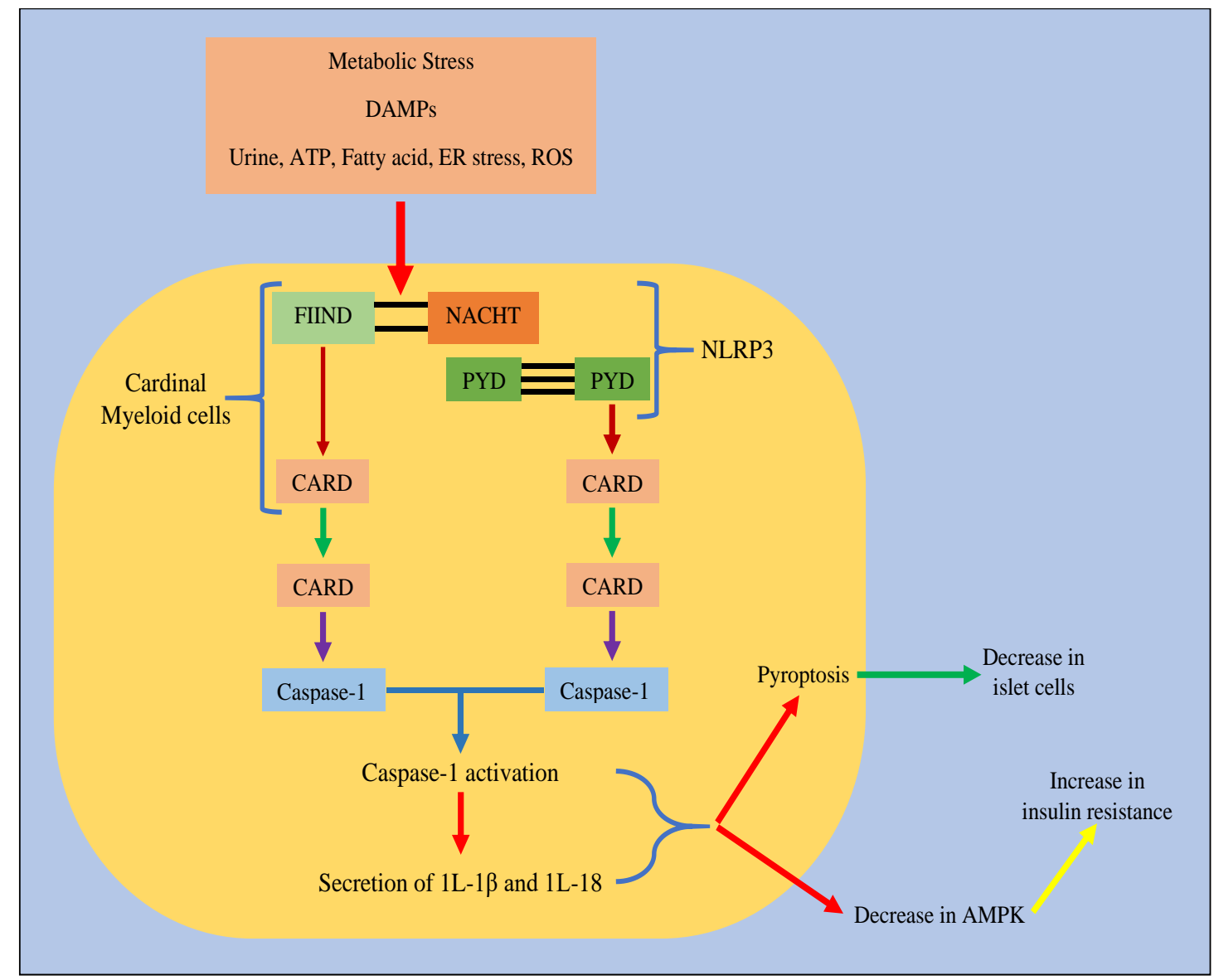

Figure 3. Role of metabolic stress in the activation of inflammasomes. 


\subsection{Nlrp3 inflammasome.}

As studied above, inflammasomes play a vital role in diabetes mellitus and are subdivided into NLRP2, NLRP3, AIM2, and NLRP4. Among them, the NLRP3 inflammasome is one of the most widely studied NLR. It also contributes to the progression of diabetes mellitus by the initiation of pyroptosis $[79,80]$. These are mainly induced by TLRs (Toll-like receptors), TNF alpha, and NF-kappa B (inflammatory cytokines). Inflammatory cytokines are necessary to produce signal-1 and signal-2 along with pro-inflammatory cytokines for further process. Signal 1 is the priming step that induces the pro-inflammatory cytokine $[81,82]$ expression, and signal-2 is an activation step triggered by PAMPs and DAMPs, thereby promoting the assembly of NLRP3 inflammasome and secretion of caspase-1 mediated proinflammatory cytokines secretion [80,82]. The main models for eliciting and activation of NLRP3 inflammasome include the ion influx model, ROS activation, and lysosome model of rupture. In the ion influx model, NLRP3 induces efflux of $\mathrm{K}^{+}$that further significantly affects the mitochondria and mtROS production. $\mathrm{K}^{+}$efflux is formed by binding of extracellular ATP and the ligand-gated ion channel P2X7R and is promoted by bacterial toxins such as; nigericin, gramicidin, and valinomycin by forming membrane pores inside the cell membrane. The link between efflux of $\mathrm{K}^{+}$and NLRP3 inflammasome activation remains unclear [83-86]. At the same time, some studies demonstrated that the efflux of $\mathrm{K}^{+}$plays a vital role in ASC upstream with the activation of NLRP3.

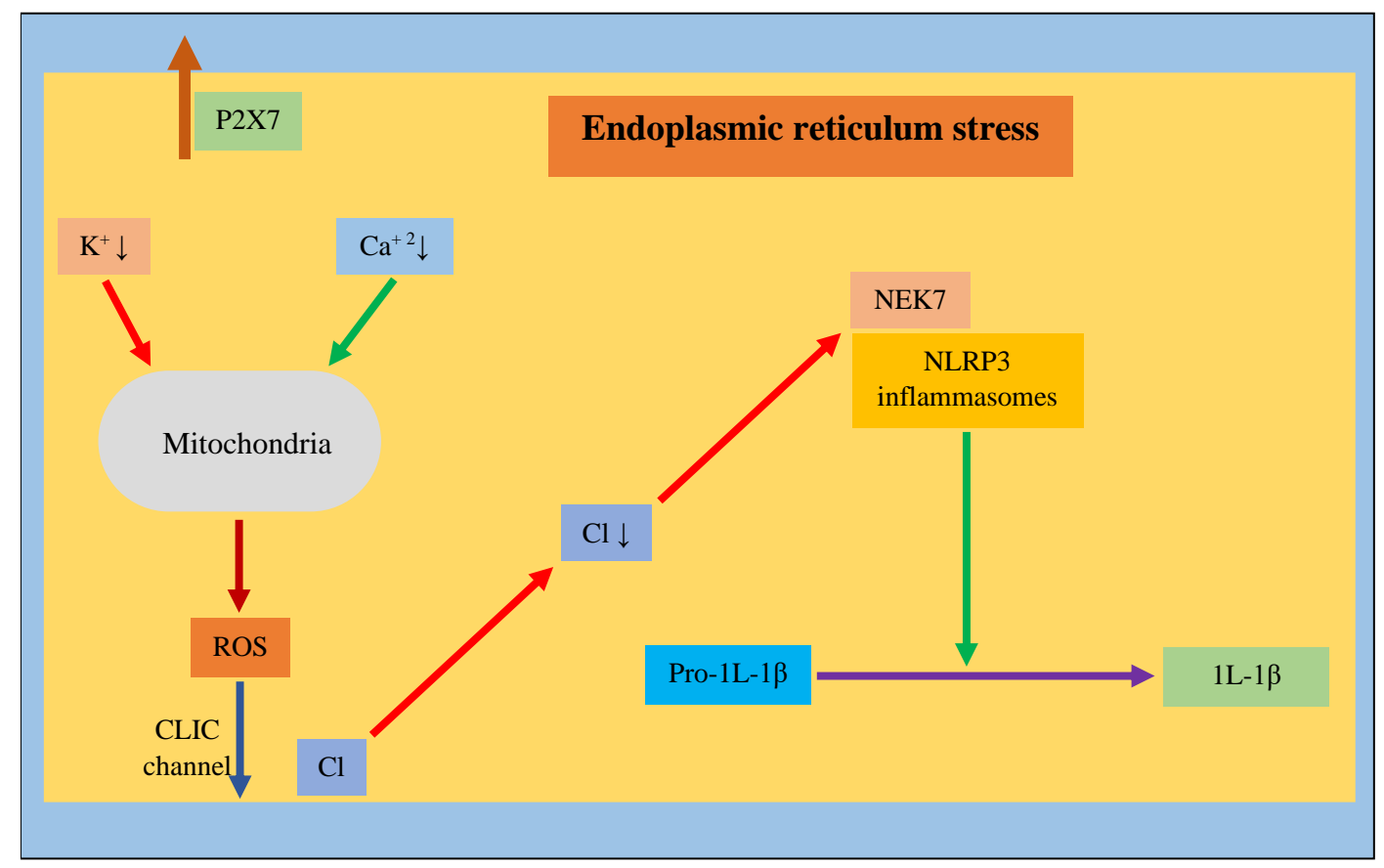

Figure 4. Role of ion in the contribution for pyroptosis.

Activation of NLRP3 can be prevented either by inhibiting $\mathrm{K}^{+}$efflux or by maintaining a high level of $\mathrm{K}^{+}$. The maximum inhibitory effect is seen when $\mathrm{K}^{+}$is $45 \mathrm{mM}$ [87-90]. These few facts regarding $\mathrm{K}^{+}$depicts that it has a fundamental role in NLRP3 activation. Though, there are some distinct perspectives regarding $\mathrm{K}^{+}$, whether it independently activates NLRP3 inflammasome or not. Some researchers contemplate that $\mathrm{K}+[91]$ is not enough to activate inflammasome alone as its activation is also related to few variations in intercellular ionic environment. Others believed that $\mathrm{K}+$ is considered the minimal event that can easily activate the NLRP3 inflammasome. Figure 4 represents the mechanism and role of $\mathrm{K}^{+}$efflux in the activation of inflammasomes. 
Damage to mitochondria stimulates the enhancement of CLCs in the cell membrane to sanction the efflux of $\mathrm{CL}^{-}[92,93]$. CL- efflux further leads to the rise in the interaction of NEK7NLRP3 and NLRP3 assembly [94-96]. Calcium released from the endoplasmic reticulum has a higher concentration than normal. This overload of $\mathrm{Ca}^{2+}$ in mitochondria additionally directs to the damage of mitochondria [97]. At last, these cause the production of mtROS, which is the most prominent cause for the activation of NLRP3 inflammasome [98]. Activation of NLRP3 inflammasome ultimately causes pyroptosis pathway by different pathways depending upon the danger signals elicited out by the disease. The diagrammatic representation shows efflux and influx of ions causing pyroptosis in diabetic patients (Figure 4).

As studied above, ions lead to the activation of inflammasome that further causes pyroptosis [99]. Likewise, pyroptosis is also caused by diabetes when the inflammasome gets activated in response to danger signals. Those signals are sensed by danger-associated molecular patterns (DAMPs) and pathogenic associated molecular patterns (PAMPs) [100]. As studied above, pyroptosis and cell lysis are regulated by caspase-1 [101] (both caspase-1dependent and caspase-1-independent having similar morphological characteristics). Both encompass cell swelling, absence of DNA laddering, condensation of chromatin, etc. The caspase-1-independent pyroptosis is only executed by human caspase-4 and 5 [102-104]. Loss in the membrane potential of mitochondria is widely attributed to cells undergoing pyroptosis. This loss ultimately causes cytoplasmic content release from the cells that encompass DAMP alarmins and ligands [105-108].

Current studies also suggested that modifications of NLRP3 inflammasome at a posttranslational level, such as phosphorylation and ubiquitination, play a vital role in its activation. It was also reported that ubiquitination of NLRP3 is seen at its LRR and NOD before its activation. Removal of these chains by a deubiquitinase, BRAC1/BRAC2 containing complex are used to activate NLRP3. Non-receptor type 22 and protein tyrosine phosphate lead to activation of this inflammasome and the release of $1 \mathrm{~L}-1 \beta$. These studies explain that NLRP3 priming is followed by either phosphorylation or by ubiquitination to regulate NLRP3 activation. This activation of NLRP3 is only seen in a person with a disorder, leading to pyroptosis. Still, further studies are needed to get clarity upon various mechanisms of NLRP3 activation [109].

\section{Conclusion}

Because of the prevalence of diabetes mellitus, cell death is a response of the body against any disease and infection. Pyroptosis, necrosis, and apoptosis are the forms of cell death. Pyroptosis is a form of programmed cell death regulated by inflammasomes that act as a defense mechanism against diabetes. Diabetes mellitus is a progressive disease associated with the depletion of beta cells that form insulin. In this review, it has been discussed that pyroptosis plays a significant role in the niche of diabetes mellitus. NLRP3 inflammasome is a type of inflammasome that activates various danger signals, ROS, DAMPs, and PAMPs and leads to the activation of caspase-1 [110]. Caspase-1, a protease, initiates the cell death process. Caspase-1 is an interleukin-1L-1beta converting enzyme, as it converts interleukin 1beta into its mature form, i.e., in mature inflammatory cytokines. Apart from Nlrp3, Gasdermin D is evolved, acting as an executioner of pyroptosis by forming pores. Both inflammasome and gasdermin D are associated with the cleavage of caspase-1, thereby leading to the rupture of the membrane. Pyroptosis has also been shown to play a vital role in diabetic complications, thus making it severe. 
In a nutshell, it can be stated that inflammasome leads to pyroptosis in diabetes patients. In the future, the microbial factors that trigger caspase- 1 activation have been taken into consideration to study those mechanisms clearly and take out the conclusion to stop the activation. The development of neo-epitope biomarkers may sense the beta loss. It may be used with the biomarkers used for diabetes evaluation [111,112].

\section{Funding}

This review received no external funding.

\section{Acknowledgments}

The authors express gratitude to Chitkara College of Pharmacy, Chitkara University, Punjab, India, for motivational support for this review's compilation.

\section{Conflict of Interest}

The authors declare no conflict of interest.

\section{References}

1. Weinlich, R.; Oberst, A.; Beere, H.M.; Green, D.R. Necroptosis in development, inflammation and disease. Nature Reviews Molecular Cell Biology 2017, 18, 127-136, https://doi.org/10.1038/nrm.2016.149.

2. Walle, L.V.; Lamkanfi, M. Pyroptosis. Curr. Biol. 2016, 26, R568-R572, https://doi.org/10.1016/j.cub.2016.02.019.

3. Frantz, S.; Ducharme, A.; Sawyer, D.; Rohde, L.E.; Kobzik, L.; Fukazawa, R.; Tracey, D.; Allen, H.; Lee, R.T.; Kelly, R.A. Targeted deletion of caspase-1 reduces early mortality and left ventricular dilatation following myocardial infarction. J. Mol. Cell. Cardiol. 2003, 35, 685-694, https://doi.org/10.1016/s00222828(03)00113-5.

4. Molla, M.D.; Akalu, Y.; Geto, Z.; Dagnew, B.; Ayelign, B.; Shibabaw, T. Role of Caspase-1 in the Pathogenesis of Inflammatory-Associated Chronic Noncommunicable Diseases. Journal of Inflammation Research 2020, 13, 749, https://doi.org/10.2147/JIR.S277457.

5. Tang, J.; Jin, L.; Liu, Y.; Li, L.; Ma, Y.; Lu, L.; Ma, J.; Ding, P.; Yang, X.; Liu, J. Exosomes Derived from Mesenchymal Stem Cells Protect the Myocardium Against Ischemia/Reperfusion Injury Through Inhibiting Pyroptosis. Drug Des. Devel. Ther. 2020, 14, 3765, https://doi.org/10.2147/DDDT.S239546.

6. Goh, G.; Ahn, M.; Zhu, F.; Lee, L.B.; Luo, D.; Irving, A.T.; Wang, L.-F. Complementary regulation of caspase- 1 and IL-1 $\beta$ reveals additional mechanisms of dampened inflammation in bats. Proceedings of the National Academy of Sciences 2020, 117, 28939, https://doi.org/10.1073/pnas.2003352117.

7. Bergsbaken, T.; Fink, S.L.; Cookson, B.T. Pyroptosis: host cell death and inflammation. Nature Reviews Microbiology 2009, 7, 99-109, https://doi.org/10.1038/nrmicro2070.

8. Fink, S.L.; Cookson, B.T. Caspase-1-dependent pore formation during pyroptosis leads to osmotic lysis of infected host macrophages. Cell. Microbiol. 2006, 8, 1812-1825, https://doi.org/10.1111/j.14625822.2006.00751.x.

9. Tsuchiya, K. Switching from Apoptosis to Pyroptosis: Gasdermin-Elicited Inflammation and Antitumor Immunity. Int. J. Mol. Sci. 2021, 22, https://doi.org/10.3390/ijms22010426.

10. Akhade, A.S.; Atif, S.M.; Lakshmi, B.S.; Dikshit, N.; Hughes, K.T.; Qadri, A.; Subramanian, N. Type 1 interferon-dependent repression of NLRC4 and iPLA2 licenses down-regulation of Salmonella flagellin inside macrophages. Proceedings of the National Academy of Sciences 2020, 117, 29811, https://doi.org/10.1073/pnas.2002747117.

11. Imre, G. Chapter Five - The involvement of regulated cell death forms in modulating the bacterial and viral pathogenesis. In Int. Rev. Cell Mol. Biol., Spetz, J.K.E., Galluzzi, L., Eds. Academic Press: 2020; Vol. 353, 211-253, https://doi.org/10.1016/bs.ircmb.2019.12.008. 
12. Kesavardhana, S.; Malireddi, R.K.S.; Kanneganti, T.-D. Caspases in Cell Death, Inflammation, and Pyroptosis. Annu. Rev. Immunol. 2020, 38, 567-595, https://doi.org/10.1146/annurev-immunol-073119095439.

13. Zhou, X.; Gordon, S.A.; Kim, Y.-M.; Hoffman, R.A.; Chen, Y.; Zhang, X.-R.; Simmons, R.L.; Ford, H.R. Nitric Oxide Induces Thymocyte Apoptosis Via a Caspase-1-Dependent Mechanism. The Journal of Immunology 2000, 165, 1252, https://doi.org/10.4049/jimmunol.165.3.1252.

14. Jorgensen, I.; Miao, E.A. Pyroptotic cell death defends against intracellular pathogens. Immunol. Rev. 2015, 265(1), 130-142, https://doi.org/10.1111/imr.12287.

15. Lasram, M.M.; Dhouib, I.B.; Annabi, A.; El Fazaa, S.; Gharbi, N. A review on the molecular mechanisms involved in insulin resistance induced by organophosphorus pesticides. Toxicology 2014, 322, 1-13, https://doi.org/10.1016/j.tox.2014.04.009.

16. Daneman, D. Type 1 diabetes. The Lancet 2006, 367, 847-858, https://doi.org/10.1016/S01406736(06)68341-4.

17. Donnelly, C.; Williams, A. Investigating the potential impact of post translational modification of autoantigens by tissue transglutaminase on humoral islet autoimmunity in type 1 diabetes. Metabolism Open 2020, 8, 100062, https://doi.org/10.1016/j.metop.2020.100062.

18. Wajchenberg, B.L. $\beta$-Cell Failure in Diabetes and Preservation by Clinical Treatment. Endocr. Rev. 2007, 28, 187-218, https://doi.org/10.1210/10.1210/er.2006-0038.

19. Cheng, C.-N.; Wang, C.-Y.; Lin, H.-W.; Chang, T.-Y.; Lin, H.-J.; Chou, C.; Lin, F.-J. Clinical outcomes of basal insulin and oral antidiabetic agents as an add-on to dual therapy in patients with type 2 diabetes mellitus. Sci. Rep. 2020, 10, 5746, https://doi.org/10.1038/s41598-020-62646-z.

20. Le Floch, J.P.; Doucet, J.; Bauduceau, B.; Verny, C.; Intergroup, S.S. Retinopathy, nephropathy, peripheral neuropathy and geriatric scale scores in elderly people with Type 2 diabetes. Diabet. Med. 2014, 31, 107111, https://doi.org/10.1111/dme.12327.

21. de Zoete, M.R.; Palm, N.W.; Zhu, S.; Flavell, R.A. Inflammasomes. Cold Spring Harb. Perspect. Biol. 2014, 6, a016287, https://doi.org/10.1101/cshperspect.a016287.

22. Balahura, L.R.; Selaru, A.; Dinescu, S.; Costache, M. Inflammation and Inflammasomes: Pros and Cons in Tumorigenesis. Journal of Immunology Research 2020, 2020, 2549763, https://doi.org/10.1155/2020/2549763.

23. Ting, J.P.Y.; Lovering, R.C.; Alnemri, E.S.; Bertin, J.; Boss, J.M.; Davis, B.K.; Flavell, R.A.; Girardin, S.E.; Godzik, A.; Harton, J.A. The NLR gene family: a standard nomenclature. Immunity 2008, 28, 285-287, https://doi.org/10.1016/j.immuni.2008.02.005.

24. Li, S.; Liang, F.; Kwan, K.; Tang, Y.; Wang, X.; Tang, Y.; Li, J.; Yang, H.; Chavan, S.S.; Wang, H.; Andersson, U.; Lu, B.; Tracey, K.J. Identification of ethyl pyruvate as a NLRP3 inflammasome inhibitor that preserves mitochondrial integrity. Mol. Med. 2018, 24, 8, https://doi.org/10.1186/s10020-018-0006-9.

25. Groslambert, M.; Py, B.F. Spotlight on the NLRP3 inflammasome pathway. Journal of inflammation research 2018, 11, 359, https://doi.org/10.2147/JIR.S141220.

26. Al Mamun, A.; Wu, Y.; Monalisa, I.; Jia, C.; Zhou, K.; Munir, F.; Xiao, J. Role of pyroptosis in spinal cord injury and its therapeutic implications. Journal of Advanced Research 2021, 28, 97-109, https://doi.org/10.1016/j.jare.2020.08.004.

27. Kelley, N.; Jeltema, D.; Duan, Y.; He, Y. The NLRP3 Inflammasome: An Overview of Mechanisms of Activation and Regulation. Int. J. Mol. Sci. 2019, 20, https://doi.org/10.3390/ijms20133328.

28. Triplitt, C.; Solis-Herrera, C.; Cersosimo, E.; Abdul-Ghani, M.; Defronzo, R.A. Empagliflozin and linagliptin combination therapy for treatment of patients with type 2 diabetes mellitus. Expert Opin. Pharmacother. 2015, 16, 2819-2833, https://doi.org/10.1517/14656566.2015.1114098.

29. Valaiyapathi, B.; Gower, B.; Ashraf, A.P. Pathophysiology of type 2 diabetes in children and adolescents. Curr. Diabetes Rev. 2020, 16, 220-229, https://doi.org/10.2174/1573399814666180608074510.

30. Atkinson, M.A.; Bluestone, J.A.; Eisenbarth, G.S.; Hebrok, M.; Herold, K.C.; Accili, D.; Pietropaolo, M.; Arvan, P.R.; Von Herrath, M.; Markel, D.S. How does type 1 diabetes develop?: the notion of homicide or $\beta$-cell suicide revisited. Diabetes 2011, 60, 1370-1379, https://doi.org/10.2337/db10-1797.

31. Michels, A.; Zhang, L.; Khadra, A.; Kushner, J.A.; Redondo, M.J.; Pietropaolo, M. Prediction and prevention of type 1 diabetes: update on success of prediction and struggles at prevention. Pediatr. Diabetes 2015, 16, 465-484, https://doi.org/10.1111/pedi.12299.

32. Nolan, C.J.; Damm, P.; Prentki, M. Type 2 diabetes across generations: from pathophysiology to prevention and management. The Lancet 2011, 378, 169-181, https://doi.org/10.1016/S0140-6736(11)60614-4. 
33. Gakidou, E.; Mallinger, L.; Abbott-Klafter, J.; Guerrero, R.; Villalpando, S.; Ridaura, R.L.; Aekplakorn, W.; Naghavi, M.; Lim, S.; Lozano, R. Management of diabetes and associated cardiovascular risk factors in seven countries: a comparison of data from national health examination surveys. Bull. W.H.O. 2011, 89, 172-183, https://doi.org/10.2471/BLT.10.080820.

34. Kahn, S.E.; Cooper, M.E.; Del Prato, S. Pathophysiology and treatment of type 2 diabetes: perspectives on the past, present, and future. The Lancet 2014, 383, 1068-1083, https://doi.org/10.1016/S01406736(13)62154-6.

35. Komada, T.; Muruve, D.A. The role of inflammasomes in kidney disease. Nature Reviews Nephrology 2019, 15, 501-520, https://doi.org/10.1038/s41581-019-0158-z.

36. Jo, E.-K.; Kim, J.K.; Shin, D.-M.; Sasakawa, C. Molecular mechanisms regulating NLRP3 inflammasome activation. Cell. Mol. Immunol. 2016, 13, 148-159, https://doi.org/10.1038/cmi.2015.95.

37. Wen, L.; Yang, H.; Ma, L.; Fu, P. The roles of NLRP3 inflammasome-mediated signaling pathways in hyperuricemic nephropathy. Mol. Cell. Biochem. 2021, https://doi.org/10.1007/s11010-020-03997-z.

38. Yin, Y.; Zhou, Z.; Liu, W.; Chang, Q.; Sun, G.; Dai, Y. Vascular endothelial cells senescence is associated with NOD-like receptor family pyrin domain-containing 3 (NLRP3) inflammasome activation via reactive oxygen species (ROS)/thioredoxin-interacting protein (TXNIP) pathway. Int. J. Biochem. Cell Biol. 2017, 84, 22-34, https://doi.org/10.1016/j.biocel.2017.01.001.

39. Surabhi, S.; Cuypers, F.; Hammerschmidt, S.; Siemens, N. The Role of NLRP3 Inflammasome in Pneumococcal Infections. Front. Immunol. 2020, 11, 3277, https://doi.org/10.3389/fimmu.2020.614801.

40. Sharma, D.; Kanneganti, T.-D. The cell biology of inflammasomes: Mechanisms of inflammasome activation and regulation. J. Cell Biol. 2016, 213, 617-629, https://doi.org/10.1083/jcb.201602089.

41. Wang, L.; Hauenstein, A.V. The NLRP3 inflammasome: Mechanism of action, role in disease and therapies. Mol. Aspects Med. 2020, 76, 100889, https://doi.org/10.1016/j.mam.2020.100889.

42. Mangan, M.S.J.; Olhava, E.J.; Roush, W.R.; Seidel, H.M.; Glick, G.D.; Latz, E. Targeting the NLRP3 inflammasome in inflammatory diseases. Nature Reviews Drug Discovery 2018, 17, 588-606, https://doi.org/10.1038/nrd.2018.97.

43. Pellegrini, C.; Antonioli, L.; Lopez-Castejon, G.; Blandizzi, C.; Fornai, M. Canonical and Non-Canonical Activation of NLRP3 Inflammasome at the Crossroad between Immune Tolerance and Intestinal Inflammation. Front. Immunol. 2017, 8, 36, https://doi.org/10.3389/fimmu.2017.00036.

44. Downs, K.P.; Nguyen, H.; Dorfleutner, A.; Stehlik, C. An overview of the non-canonical inflammasome. Mol. Aspects Med. 2020, 76, 100924, https://doi.org/10.1016/j.mam.2020.100924.

45. Shi, J.; Zhao, Y.; Wang, Y.; Gao, W.; Ding, J.; Li, P.; Hu, L.; Shao, F. Inflammatory caspases are innate immune receptors for intracellular LPS. Nature 2014, 514, 187-192, https://doi.org/10.1038/nature13683.

46. Kayagaki, N.; Stowe, I.B.; Lee, B.L.; O’Rourke, K.; Anderson, K.; Warming, S.; Cuellar, T.; Haley, B.; Roose-Girma, M.; Phung, Q.T.; Liu, P.S.; Lill, J.R.; Li, H.; Wu, J.; Kummerfeld, S.; Zhang, J.; Lee, W.P.; Snipas, S.J.; Salvesen, G.S.; Morris, L.X.; Fitzgerald, L.; Zhang, Y.; Bertram, E.M.; Goodnow, C.C.; Dixit, V.M. Caspase-11 cleaves gasdermin D for non-canonical inflammasome signalling. Nature 2015, 526, 666671, https://doi.org/10.1038/nature15541.

47. Kayagaki, N.; Warming, S.; Lamkanfi, M.; Walle, L.V.; Louie, S.; Dong, J.; Newton, K.; Qu, Y.; Liu, J.; Heldens, S.; Zhang, J.; Lee, W.P.; Roose-Girma, M.; Dixit, V.M. Non-canonical inflammasome activation targets caspase-11. Nature 2011, 479, 117-121, https://doi.org/10.1038/nature10558.

48. Eren, E.; Planès, R.; Bagayoko, S.; Bordignon, P.-J.; Chaoui, K.; Hessel, A.; Santoni, K.; Pinilla, M.; Lagrange, B.; Burlet-Schiltz, O.; Howard, J.C.; Henry, T.; Yamamoto, M.; Meunier, E. Irgm2 and Gate-16 cooperatively dampen Gram-negative bacteria-induced caspase-11 response. EMBO reports 2020, 21, e50829, https://doi.org/10.15252/embr.202050829.

49. Zhang, L.; Jiang, X.; Pfau, D.; Ling, Y.; Nathan, C.F. Type I interferon signaling mediates Mycobacterium tuberculosis-induced macrophage death. J. Exp. Med. 2020, 218, https://doi.org/10.1084/jem.20200887.

50. Cerqueira, D.M.; Pereira, M.S.; Silva, A.L.; Cunha, L.D.; Zamboni, D.S. Caspase-1 but not caspase-11 is required for NLRC4-mediated pyroptosis and restriction of infection by flagellated Legionella species in mouse macrophages and in vivo. J. Immunol. 2015, 195(5), 2303-2311, https://doi.org/10.4049/jimmunol.1501223.

51. Van Opdenbosch, N.; Lamkanfi, M. Caspases in cell death, inflammation, and disease. Immunity 2019, 50(6), 1352-1364, https://doi.org/10.1016/j.immuni.2019.05.020. 
52. Shi, J.; Zhao, Y.; Wang, K.; Shi, X.; Wang, Y.; Huang, H.; Zhuang, Y.; Cai, T.; Wang, F.; Shao, F. Cleavage of GSDMD by inflammatory caspases determines pyroptotic cell death. Nature 2015, 526, 660-665, https://doi.org/10.1038/nature15514.

53. Miao, E.A.; Leaf, I.A.; Treuting, P.M.; Mao, D.P.; Dors, M.; Sarkar, A.; Warren, S.E.; Wewers, M.D.; Aderem, A. Caspase-1-induced pyroptosis is an innate immune effector mechanism against intracellular bacteria. Nat. Immunol. 2010, 11, 1136-1142, https://doi.org/10.1038/ni.1960.

54. Demarco, B.; Grayczyk, J.P.; Bjanes, E.; Le Roy, D.; Tonnus, W.; Assenmacher, C.-A.; Radaelli, E.; Fettrelet, T.; Mack, V.; Linkermann, A.; Roger, T.; Brodsky, I.E.; Chen, K.W.; Broz, P. Caspase-8dependent gasdermin D cleavage promotes antimicrobial defense but confers susceptibility to TNF-induced lethality. Science Advances 2020, 6, eabc3465, https://doi.org/10.1126/sciadv.abc3465.

55. Miao, E.A.; Rajan, J.V.; Aderem, A. Caspase-1-induced pyroptotic cell death. Immunol. Rev. 2011, 243 , 206-214, https://doi.org/10.1111/j.1600-065X.2011.01044.X.

56. Orning, P.; Lien, E.; Fitzgerald, K.A. Gasdermins and their role in immunity and inflammation. J. Exp. Med. 2019, 216, 2453-2465, https://doi.org/10.1084/jem.20190545.

57. Yang, Y.; Wang, H.; Kouadir, M.; Song, H.; Shi, F. Recent advances in the mechanisms of NLRP3 inflammasome activation and its inhibitors. Cell Death Dis. 2019, 10, 128, https://doi.org/10.1038/s41419019-1413-8.

58. Xia, S.; Hollingsworth, L.R.; Wu, H. Mechanism and regulation of gasdermin-mediated cell death. Cold Spring Harb. Perspect. Biol. 2020, 12, a036400, https://doi.org/10.1101/cshperspect.a036400.

59. Rogers, C.; Fernandes-Alnemri, T.; Mayes, L.; Alnemri, D.; Cingolani, G.; Alnemri, E.S. Cleavage of DFNA5 by caspase-3 during apoptosis mediates progression to secondary necrotic/pyroptotic cell death. Nature Communications 2017, 8, 14128, https://doi.org/10.1038/ncomms14128.

60. Liu, X.; Zhang, Z.; Ruan, J.; Pan, Y.; Magupalli, V.G.; Wu, H.; Lieberman, J. Inflammasome-activated gasdermin D causes pyroptosis by forming membrane pores. Nature 2016, 535, 153-158, https://doi.org/10.1038/nature18629.

61. Fan, G.; Li, Y.; Chen, J.; Zong, Y.; Yang, X. DHA/AA alleviates LPS-induced Kupffer cells pyroptosis via GPR120 interaction with NLRP3 to inhibit inflammasome complexes assembly. Cell Death Dis. 2021, 12, 73, https://doi.org/10.1038/s41419-020-03347-3.

62. Platnich, J.M.; Chung, H.; Lau, A.; Sandall, C.F.; Bondzi-Simpson, A.; Chen, H.M.; Komada, T.; TrotmanGrant, A.C.; Brandelli, J.R.; Chun, J.; Beck, P.L. Shiga toxin/lipopolysaccharide activates caspase-4 and gasdermin D to trigger mitochondrial reactive oxygen species upstream of the NLRP3 inflammasome. Cell Rep. 2018, 25(6), 1525-1536, https://doi.org/10.1016/j.celrep.2018.09.071.

63. Ding, J.; Wang, K.; Liu, W.; She, Y.; Sun, Q.; Shi, J.; Sun, H.; Wang, D.-C.; Shao, F. Pore-forming activity and structural autoinhibition of the gasdermin family. Nature 2016, 535, 111-116, https://doi.org/10.1038/nature18590.

64. Tamura, M.; Tanaka, S.; Fujii, T.; Aoki, A.; Komiyama, H.; Ezawa, K.; Sumiyama, K.; Sagai, T.; Shiroishi, T. Members of a novel gene family, Gsdm, are expressed exclusively in the epithelium of the skin and gastrointestinal tract in a highly tissue-specific manner. Genomics 2007, 89, 618-629, https://doi.org/10.1016/j.ygeno.2007.01.003.

65. Sborgi, L.; Rühl, S.; Mulvihill, E.; Pipercevic, J.; Heilig, R.; Stahlberg, H.; Farady, C.J.; Müller, D.J.; Broz, P.; Hiller, S. GSDMD membrane pore formation constitutes the mechanism of pyroptotic cell death. The EMBO Journal 2016, 35, 1766-1778, https://doi.org/10.15252/embj.201694696.

66. Kovacs, S.B.; Miao, E.A. Gasdermins: effectors of pyroptosis. Trends Cell Biol. 2017, 27, 673-684, https://doi.org/10.1016/j.tcb.2017.05.005.

67. Pandeya, A.; Li, L.; Li, Z.; Wei, Y. Gasdermin D (GSDMD) as a new target for the treatment of infection. MedChemComm 2019, 10, 660-667, https://doi.org/10.1039/C9MD00059C.

68. Zuo, L.; Zhou, L.; Wu, C.; Wang, Y.; Li, Y.; Huang, R.; Wu, S. Salmonella spvC Gene Inhibits Pyroptosis and Intestinal Inflammation to Aggravate Systemic Infection in Mice. Front. Microbiol. 2020, 11, 3135 , https://doi.org/10.3389/fmicb.2020.562491.

69. Hoffmann, E.K.; Lambert, I.H.; Pedersen, S.F. Physiology of Cell Volume Regulation in Vertebrates. Physiol. Rev. 2009, 89, 193-277, https://doi.org/10.1152/physrev.00037.2007.

70. McNeil, P.L.; Kirchhausen, T. An emergency response team for membrane repair. Nature Reviews Molecular Cell Biology 2005, 6, 499-505, https://doi.org/10.1038/nrm1665. 
71. van den Oever, I.A.M.; Raterman, H.G.; Nurmohamed, M.T.; Simsek, S. Endothelial Dysfunction, Inflammation, and Apoptosis in Diabetes Mellitus. Mediators Inflamm. 2010, 2010, 792393, https://doi.org/10.1155/2010/792393.

72. Zhang, P.; Li, T.; Wu, X.; Nice, E.C.; Huang, C.; Zhang, Y. Oxidative stress and diabetes: antioxidative strategies. Front. Med. 2020, 14, 583-600, https://doi.org/10.1007/s11684-019-0729-1.

73. Yang, Z.; Mo, X.; Gong, Q.; Pan, Q.; Yang, X.; Cai, W.; Li, C.; Ma, J.-x.; He, Y.; Gao, G. Critical effect of VEGF in the process of endothelial cell apoptosis induced by high glucose. Apoptosis 2008, 13, 1331-1343, https://doi.org/10.1007/s10495-008-0257-y.

74. Luc, K.; Schramm-Luc, A.; Guzik, T.J.; Mikolajczyk, T.P. Oxidative stress and inflammatory markers in prediabetes and diabetes. J. Physiol. Pharmacol 2019, 70, 809-824, https://doi.org/10.26402/jpp.2019.6.01.

75. Rendra, E.; Riabov, V.; Mossel, D.M.; Sevastyanova, T.; Harmsen, M.C.; Kzhyshkowska, J. Reactive oxygen species (ROS) in macrophage activation and function in diabetes. Immunobiology 2019, 224, 242253, https://doi.org/10.1016/j.imbio.2018.11.010.

76. Grant, R.; Dixit, V. Mechanisms of disease: inflammasome activation and the development of type 2 diabetes. Front. Immunol. 2013, 4, 50, https://doi.org/10.3389/fimmu.2013.00050.

77. Dixit, V.D. Nlrp3 inflammasome activation in type 2 diabetes: is it clinically relevant? Diabetes 2013, 62, 22-24, https://doi.org/10.2337/db12-1115.

78. Zhou, R.; Tardivel, A.; Thorens, B.; Choi, I.; Tschopp, J. Thioredoxin-interacting protein links oxidative stress to inflammasome activation. Nat. Immunol. 2010, 11, 136-140, https://doi.org/10.1038/ni.1831.

79. Yeon, S.H.; Yang, G.; Lee, H.E.; Lee, J.Y. Oxidized phosphatidylcholine induces the activation of NLRP3 inflammasome in macrophages. J. Leukoc. Biol. 2017, 101(1), 205-215, https://doi.org/10.1189/jlb.3VMA1215-579RR.

80. Yu, Z.-W.; Zhang, J.; Li, X.; Wang, Y.; Fu, Y.-H.; Gao, X.-Y. A new research hot spot: The role of NLRP3 inflammasome activation, a key step in pyroptosis, in diabetes and diabetic complications. Life Sci. 2020, 240, 117138, https://doi.org/10.1016/j.lfs.2019.117138.

81. Pereira, C.A.; Carlos, D.; Ferreira, N.S.; Silva, J.F.; Zanotto, C.Z.; Zamboni, D.S.; Garcia, V.D.; Ventura, D.F.; Silva, J.S.; Tostes, R.C. Mitochondrial DNA Promotes NLRP3 Inflammasome Activation and Contributes to Endothelial Dysfunction and Inflammation in Type 1 Diabetes. Front. Physiol. 2020, 10, 1557, https://doi.org/10.3389/fphys.2019.01557.

82. Guarda, G.; Zenger, M.; Yazdi, A.S.; Schroder, K.; Ferrero, I.; Menu, P.; Tardivel, A.; Mattmann, C.; Tschopp, J. Differential expression of NLRP3 among hematopoietic cells. The Journal of Immunology 2011, 186, 2529-2534, https://doi.org/10.4049/jimmunol.1002720.

83. Bai, B.; Yang, Y.; Wang, Q.; Li, M.; Tian, C.; Liu, Y.; Aung, L.H.H.; Li, P.-f.; Yu, T.; Chu, X.-m. NLRP3 inflammasome in endothelial dysfunction. Cell Death Dis. 2020, 11, 776, https://doi.org/10.1038/s41419020-02985-x.

84. Mariathasan, S.; Weiss, D.S.; Newton, K.; McBride, J.; O'Rourke, K.; Roose-Girma, M.; Lee, W.P.; Weinrauch, Y.; Monack, D.M.; Dixit, V.M. Cryopyrin activates the inflammasome in response to toxins and ATP. Nature 2006, 440, 228-232, https://doi.org/10.1038/nature04515.

85. Rühl, S.; Broz, P. Caspase-11 activates a canonical NLRP3 inflammasome by promoting K+ efflux. Eur. J. Immunol. 2015, 45, 2927-2936, https://doi.org/10.1002/eji.201545772.

86. Islam, M.T.; Bardaweel, S.K.; Mubarak, M.S.; Koch, W.; Gaweł-Beben, K.; Antosiewicz, B.; Sharifi-Rad, J. Immunomodulatory Effects of Diterpenes and Their Derivatives Through NLRP3 Inflammasome Pathway: A Review. Front. Immunol. 2020, 11, 2234, https://doi.org/10.3389/fimmu.2020.572136.

87. Muñoz-Planillo, R.; Kuffa, P.; Martínez-Colón, G.; Smith, B.L.; Rajendiran, T.M.; Núñez, G. K+ efflux is the common trigger of NLRP3 inflammasome activation by bacterial toxins and particulate matter. Immunity 2013, 38, 1142-1153, https://doi.org/10.1016/j.immuni.2013.05.016.

88. Xu, Z.; Chen, Z.-m.; Wu, X.; Zhang, L.; Cao, Y.; Zhou, P. Distinct Molecular Mechanisms Underlying Potassium Efflux for NLRP3 Inflammasome Activation. Front. Immunol. 2020, 11, 3207, https://doi.org/10.3389/fimmu.2020.609441.

89. Zhou, Y.; Tong, Z.; Jiang, S.; Zheng, W.; Zhao, J.; Zhou, X. The Roles of Endoplasmic Reticulum in NLRP3 Inflammasome Activation. Cells 2020, 9, https://doi.org/10.3390/cells9051219.

90. Leu, W.-J.; Chu, J.-C.; Hsu, J.-L.; Du, C.-M.; Jiang, Y.-H.; Hsu, L.-C.; Huang, W.-J.; Guh, J.-H. Chalcones Display Anti-NLRP3 Inflammasome Activity in Macrophages through Inhibition of Both Priming and Activation Steps-Structure-Activity-Relationship and Mechanism Studies. Molecules 2020, 25, 5960, https://doi.org/10.3390/molecules25245960. 
91. Sun, X.; Pang, H.; Li, J.; Luo, S.; Huang, G.; Li, X.; Xie, Z.; Zhou, Z. The NLRP3 Inflammasome and Its Role in T1DM. Front. Immunol. 2020, 11, 1595, https://doi.org/10.3389/fimmu.2020.01595.

92. Zheng, D.; Liwinski, T.; Elinav, E. Inflammasome activation and regulation: toward a better understanding of complex mechanisms. Cell Discov. 2020, 6(1), 1-22, https://doi.org/10.1038/s41421-020-0167-x.

93. Zhang, H.; Zahid, A.; Ismail, H.; Tang, Y.; Jin, T.; Tao, J. An overview of disease models for NLRP3 inflammasome over-activation. Expert Opinion on Drug Discovery 2020, 1-18, https://doi.org/10.1080/17460441.2021.1844179.

94. Hafner-Bratkovič, I.; Pelegrín, P. Ion homeostasis and ion channels in NLRP3 inflammasome activation and regulation. Curr. Opin. Immunol. 2018, 52, 8-17, https://doi.org/10.1016/j.coi.2018.03.010.

95. Wang, S.; Yuan, Y.-H.; Chen, N.-H.; Wang, H.-B. The mechanisms of NLRP3 inflammasome/pyroptosis activation and their role in Parkinson's disease. Int. Immunopharmacol. 2019, 67, 458-464, https://doi.org/10.1016/j.intimp.2018.12.019.

96. Yabal, M.; Calleja, D.J.; Simpson, D.S.; Lawlor, K.E. Stressing out the mitochondria: Mechanistic insights into NLRP3 inflammasome activation. J. Leukocyte Biol. 2019, 105, 377-399, https://doi.org/10.1002/JLB.MR0318-124R.

97. Clapham, D.E. TRP channels as cellular sensors. Nature 2003, 426, 517-524, https://doi.org/10.1038/nature02196.

98. Stowe, I.; Lee, B.; Kayagaki, N. Caspase-11: arming the guards against bacterial infection. Immunol. Rev. 2015, 265, 75-84, https://doi.org/10.1111/imr.12292.

99. Xu, B.; Jiang, M.; Chu, Y.; Wang, W.; Chen, D.; Li, X.; Zhang, Z.; Zhang, D.; Fan, D.; Nie, Y. Gasdermin D plays a key role as a pyroptosis executor of non-alcoholic steatohepatitis in humans and mice. J. Hepatol. 2018, 68, 773-782, https://doi.org/10.1016/j.jhep.2017.11.040.

100. Shahzad, K.; Bock, F.; Al-Dabet, M.d.M.; Gadi, I.; Kohli, S.; Nazir, S.; Ghosh, S.; Ranjan, S.; Wang, H.; Madhusudhan, T.; Nawroth, P.P.; Isermann, B. Caspase-1, but Not Caspase-3, Promotes Diabetic Nephropathy. J. Am. Soc. Nephrol. 2016, 27, 2270, https://doi.org/10.1681/ASN.2015060676.

101. Fink, S.L.; Bergsbaken, T.; Cookson, B.T. Anthrax lethal toxin and Salmonella elicit the common cell death pathway of caspase-1- dependent pyroptosis via distinct mechanisms. Proceedings of the National Academy of Sciences 2008, 105, 4312, https://doi.org/10.1073/pnas.0707370105.

102. Thurston, T.L.; Matthews, S.A.; Jennings, E.; Alix, E.; Shao, F.; Shenoy, A.R.; Birrell, M.A.; Holden, D.W. Growth inhibition of cytosolic Salmonella by caspase-1 and caspase-11 precedes host cell death. Nat. Commun. 2016, 7(1), 1-5, https://doi.org/10.1038/ncomms13292.

103. Oh, S.; Son, M.; Park, C.-H.; Jang, J.T.; Son, K.H.; Byun, K. The Reducing Effects of PyrogallolPhloroglucinol-6,6-Bieckol on High-Fat Diet-Induced Pyroptosis in Endothelial and Vascular Smooth Muscle Cells of Mice Aortas. Mar. Drugs 2020, 18, https://doi.org/10.3390/md18120648.

104. Fernandes-Alnemri, T.; Wu, J.; Yu, J.W.; Datta, P.; Miller, B.; Jankowski, W.; Rosenberg, S.; Zhang, J.; Alnemri, E.S. The pyroptosome: a supramolecular assembly of ASC dimers mediating inflammatory cell death via caspase-1 activation. Cell Death Differ. 2007, 14, 1590-1604, https://doi.org/10.1038/sj.cdd.4402194.

105. Tsuchiya, K. Inflammasome-associated cell death: Pyroptosis, apoptosis, and physiological implications. Microbiol. Immunol. 2020, 64, 252-269, https://doi.org/10.1111/1348-0421.12771.

106. Lamkanfi, M.; Dixit, V.M. Mechanisms and functions of inflammasomes. Cell 2014, 157(5), 1013-1022, https://doi.org/10.1016/j.cell.2014.04.007.

107. Shimada, K.; Crother, T.R.; Karlin, J.; Dagvadorj, J.; Chiba, N.; Chen, S.; Ramanujan, V.K.; Wolf, A.J.; Vergnes, L.; Ojcius, D.M. Oxidized mitochondrial DNA activates the NLRP3 inflammasome during apoptosis. Immunity 2012, 36, 401-414, https://doi.org/10.1016/j.immuni.2012.01.009.

108. Mariathasan, S.; Newton, K.; Monack, D.M.; Vucic, D.; French, D.M.; Lee, W.P.; Roose-Girma, M.; Erickson, S.; Dixit, V.M. Differential activation of the inflammasome by caspase-1 adaptors ASC and Ipaf. Nature 2004, 430, 213-218, https://doi.org/10.1038/nature02664.

109. Lee, H.M.; Kim, J.J.; Kim, H.J.; Shong, M.; Ku, B.J.; Jo, E.K. Upregulated NLRP3 inflammasome activation in patients with type 2 diabetes. Diabetes 2013, 62(1), 194-204, https://doi.org/10.2337/db12-0420.

110. Py, B.F.; Kim, M.-S.; Vakifahmetoglu-Norberg, H.; Yuan, J. Deubiquitination of NLRP3 by BRCC3 critically regulates inflammasome activity. Mol. Cell 2013, 49, 331-338, https://doi.org/10.1016/j.molcel.2012.11.009.

111. Ding, S.; Xu, S.; Ma, Y.; Liu, G.; Jang, H.; Fang, J. Modulatory mechanisms of the NLRP3 inflammasomes in diabetes. Biomolecules 2019, 9(12), 850, https://doi.org/10.3390/biom9120850. 
112. Neutzsky-Wulff, A.V.; Andreassen, K.V.; Hjuler, S.T.; Feigh, M.; Bay-Jensen, A.-C.; Zheng, Q.; Henriksen, K.; Karsdal, M.A. Future detection and monitoring of diabetes may entail analysis of both $\beta$-cell function and volume: How markers of $\beta$-cell loss may assist. J. Transl. Med. 2012, 10, 214, https://doi.org/10.1186/1479-5876-10-214. 